\title{
Nuevas relaciones industria residencia en municipios de escala intermedia. El caso de Beniparrell en l'Horta Sud de Valencia.
}

\author{
Julia Deltoro-Soto ${ }^{1}$, Francisco-Juan Martínez-Pérez ${ }^{2}$, \\ Carmen Blasco-Sánchez ${ }^{3}$, Ana Gascón-Hernández ${ }^{4}$ \\ ${ }^{1}$ Departamento de Ingeniería de la Construcción y de Proyectos de Ingeniería Civil. \\ ${ }^{23}$ Departamento de Urbanismo. ${ }^{123}{ }^{4}$ Universidad Politécnica de Valencia. Valencia, España. \\ E-mail: 1judelso@cst.upv.es, ${ }^{2}$ fjmartpe@urb.upv.es, \\ ${ }^{3}$ mblasco@urb.upv.es, ${ }^{4}$ angasher@doctor.upv.es
}

\begin{abstract}
Resumen. La aparición de las actividades industriales en las ciudades supuso una transformación de sus dinámicas urbanas. Alojada la industria en el interior de la ciudad, los conflictos urbanos hicieron rápidamente acto de presencia. La solución adoptada fue la relocalización de la industria fuera de los centros urbanos, alojándose en nuevos espacios especificos localizados en ámbitos territoriales segregados. Desde las últimas décadas del siglo XX hasta la actualidad, las áreas industriales han sufrido importantes transformaciones, tanto tecnológicas como de nuevos usos. Además, es habitual que la distancia que separaba los nuevos asentamientos para la industria de la ciudad de origen se haya ocupado con nuevas extensiones urbanas, lo que al final se traduce en la existencia de multitud de casos donde los espacios residenciales $e$ industriales son contiguos y configuran una entidad urbana sobre la que es necesario reflexionar, tanto en la escala municipal como en la supramunicipal. Las nuevas relaciones entre tejidos de diferente morfología, entre usos más o menos compatibles, las exigencias y nuevas pautas de la movilidad y los vínculos entre trabajo, residencia y ocio hacen necesario replantear este nuevo contexto. El caso del municipio valenciano de Beniparrell es un ejemplo paradigmático. Se trata de un municipio con menos de 2.000 habitantes, una superficie de 3'7 $\mathrm{km} 2$, de los cuales el área residencial ocupa 0'32 $\mathrm{km} 2$ y el área vinculada a la actividad económica 1,34 km2. La disposición contigua del suelo económico respecto al espacio residencial y su peso territorial plantea nuevas relaciones que exigen interpretar los tejidos como complementarios, asumiendo que los ciudadanos participan de su economía, de su paisaje y de su vitalidad de forma cotidiana.
\end{abstract}

Palabras clave: Ciudad industrial, suelo industrial, planificación urbanistica, tejido urbano

\section{Introducción}

El suelo urbano ha sido motivo de estudio por parte de urbanistas y otras profesiones que han tratado de dar soluciones para mejorar el entorno y la calidad de vida de la población. La mayoría de estos esfuerzos se han centrado históricamente en las áreas residenciales más que en las áreas industriales y en nombradas ocasiones cuando se ha realizado, han sido las cuestiones económicas las que han centrado el interés, dejando de lado las relacionadas con su organización espacial y funcional y su relación con el entorno y la ciudad.

La calidad de este suelo industrial no responde a la importancia de este suelo por su extensión y por la utilización del mismo por parte de una gran cantidad de población que pasa la mayor parte de su jornada en él. La problemática de estos suelos no se limita al mantenimiento de

http://dx.doi.org/10.4995/ISUFh2019.2019.9718

(c) EY-NC-ND 2019, Editorial Universitat Politècnica de València 
sus instalaciones y edificación, sino que es más amplia, ya que carece de servicios mínimos básicos, unas relaciones satisfactorias con las ciudades cercanas, o una forma de transporte sostenible que las comunique con las áreas residenciales, entre otras. Hasta el momento, las iniciativas y referencias que analizan esta situación y proponen soluciones han sido escasas, ya que no se han entendido como prioritarias las acciones de mantenimiento, renovación o integración de estas áreas. Con la aparición en la Comunidad Valenciana de la Ley 14 del 5 de junio de 2018, de gestión, modernización y promoción de las áreas industriales de la Comunitat Valenciana, se espera que se inicien acciones que revitalicen las áreas industriales y que además se mejoren sus relaciones con las áreas residenciales, de las que en su inicio formaron parte.

La aparición de las actividades industriales en las ciudades, con un mayor tamaño y más contaminantes que las tradicionales, supuso una transformación de sus dinámicas urbanas. Alojada la industria en los cascos urbanos los conflictos hicieron rápidamente acto de presencia, creando problemas de salud física y hacinamiento.

Posteriormente, la solución fue la relocalización de la producción industrial fuera de los ámbitos urbanos donde se construyeron fábricas con unas mejores condiciones, mayores dimensiones y más iluminadas y salubres, alojándose en nuevos espacios específicos para el nuevo uso en ámbitos territoriales segregados, (De Terán, 1972) en enclaves territoriales periféricos o periurbanos de cierta especialización y creando un nuevo paisaje singular, entornos industriales, desarrollados junto a grandes vías de comunicación (Benito, 2005), entre núcleos urbanos importantes, o bien cercanos a otras áreas como los puertos.

Los vacíos urbanos creados tras la demolición de viejas fábricas dentro de las ciudades han sido utilizados por otros usos urbanos, residencial o terciario. En otras ocasiones, debido a la importancia histórica, arquitectónica o constructiva de ciertas industrias, estos edificios se han conservado e incluso protegido, remodelándose para adaptarse a y alojar otros usos dentro de ellas.

Desde los años 60 hasta la actualidad, se están produciendo importantes transformaciones, tanto tecnológicas como de nuevos usos, y estas áreas industriales acogen actividades terciarias $\mathrm{y}$ de ocio, que hasta ahora se formalizaban dentro de la ciudad. Por ello, parece necesario repensar el concepto clásico de polígono industrial y adoptar un nuevo término como el de áreas o sectores de actividad económica, que refleje mejor la realidad que ya está consolidada en muchos lugares.

En algunos municipios, generalmente de reducida extensión, la distancia que separaba los dos tejidos ha sido completada en la actualidad. En otros casos, en vez de producirse la evolución industrial relatada previamente, no se instaló industria dentro de la ciudad y posteriormente ha aparecido directamente en su periferia. Así, las relaciones que existen con otros usos, residencial o servicios, pese a su contigüidad, son escasas o nulas. Esta dinámica se produjo en aquellos municipios que no poseían la entidad suficiente para localizar industria dentro de ellos, debido al reducido tamaño del municipio o su escasa importancia, pero que posteriormente, por su localización estratégica al situarse cercanos a rutas comerciales o infraestructuras de transporte, acogen en su término municipal distintas áreas industriales. Este es el caso de varios de los municipios del sur de Valencia, que localizados en torno al eje viario que conectaba Valencia con Alicante, desarrollaron áreas industriales desde los años 50.

La realidad actual refleja la existencia de multitud de casos donde los espacios residenciales e industriales son contiguos y configuran una entidad urbana sobre la que es necesario reflexionar, tanto en la escala municipal como en la supramunicipal. Las nuevas relaciones entre los tejidos, o la inexistencia de relación entre ellos, la existencia de usos incompatibles o la necesaria inclusión de otros usos compatibles dentro del uso industrial, las condiciones nuevas de la movilidad y los nuevos parámetros entre trabajo, residencia y ocio hacen necesario replantear el nuevo contexto. La disposición del suelo económico respecto al espacio residencial hace necesario asumir nuevas relaciones e interpretar los tejidos como complementarios, asumiendo que los ciudadanos participan de 

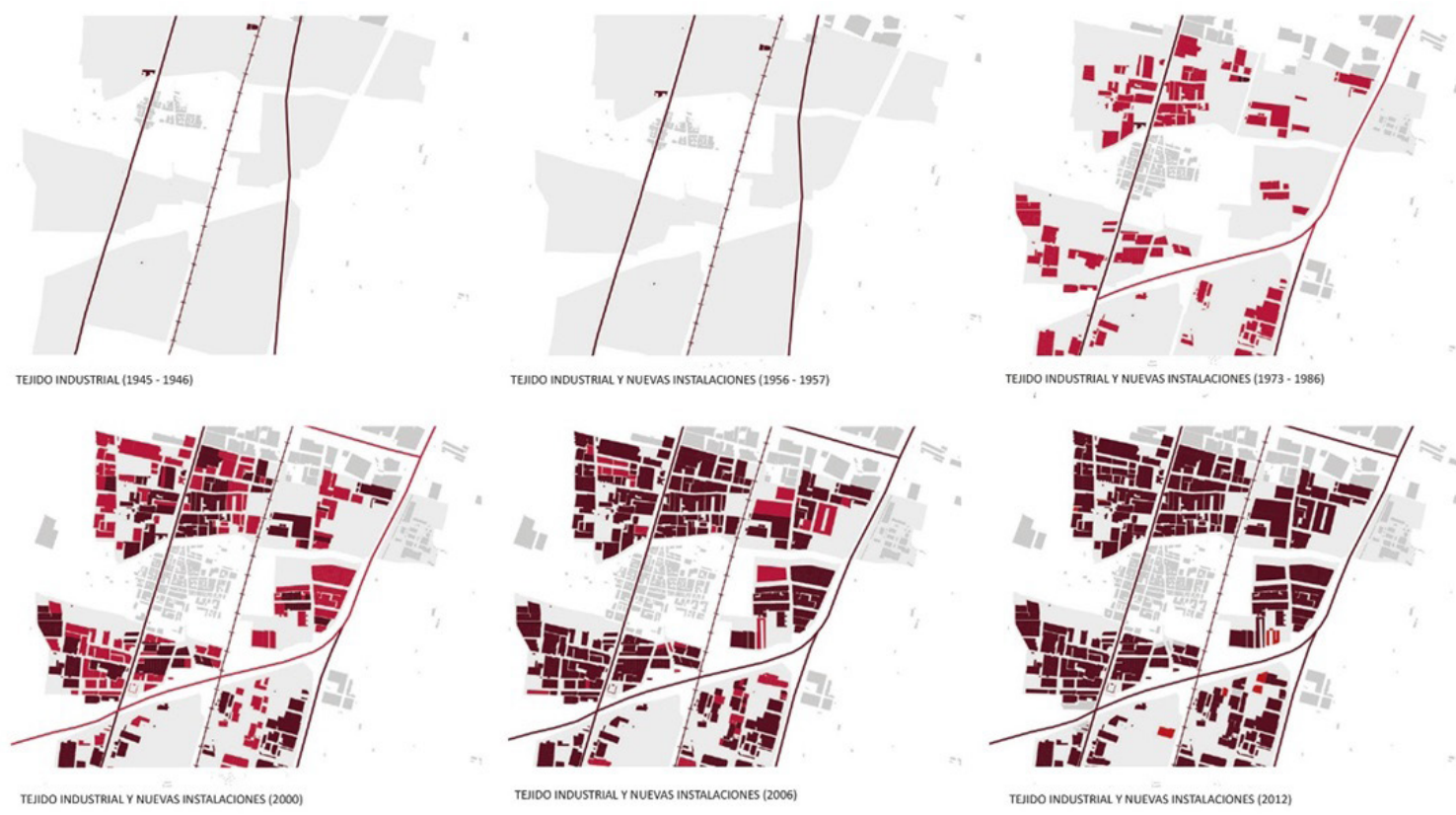

TElOO INDUSTRIAL Y NUEVVA INSTALACIONES 201

Fig. 1. Evolución histórica (Fuente: (Cano Hurtado et alt. 2018))

ambos de forma cotidiana.

\section{Beniparrell: evolución}

El núcleo urbano de Beniparrell tiene su origen en el Camí Real, vía histórica del siglo XVIII que unía Valencia y Madrid, paralelo al cual se desarrolló también la vía férrea ValenciaXàtiva a partir del XIX. Como la mayoría de los municipios de la zona, la agricultura fue su principal actividad, y no sería hasta los años 50 cuando, al igual que en el resto de territorios valencianos, se iniciaría la industrialización en su entorno. Como se puede apreciar en la Figura 1, el salto en cuanto a edificios industriales instalados se produce en los años 60, 70 y 80 junto al Camí Real o nacional 340, siguiendo las mismas dinámicas que en los municipios vecinos. Se utilizaban los viarios más importantes para ubicarse junto a ellos, resolviendo problemas de accesibilidad y aprovechando el efecto escaparate que suponía la proximidad a la vía. Sin embargo, las instalaciones se ubican en parcelarios agrícolas, sin una urbanización de viarios y manzanas, y con caminos rurales como accesos a estos edificios en muchos casos.

Estas localizaciones espontáneas dan lugar en los años 80 a un tejido industrial desorganizado, sin articulación y sin regulación urbanística, tendencia de crecimiento que se seguirá hasta la actualidad. La construcción de las áreas industriales del municipio se desarrolla principalmente entre los años 1955 y 1993 en torno al Camí Reial y en áreas muy cercanas al núcleo residencial, llegándose a colmatar el suelo ya en 2005. En esta etapa, industria y núcleo residencial siguen estando separadas espacialmente, al norte por el límite natural del barranco, al sur por terrenos agrícolas y al oeste por la vía del tren, siendo las barreras físicas o naturales las que producen esa separación entre el uso industrial y residencial (zoning) que pretende segregar los usos incompatibles y prevenir riesgos ambientales (Hatuka 2017). En este periodo ya es significativa la magnitud de suelo industrial edificado en relación con el suelo residencial del casco urbano.

La importancia territorial de las vías de comunicación que atraviesan el municipio, así como la construcción de la V-21 harán que el eje sur de Valencia se consolide como eje industrial. En la ortofoto del 2000 se puede observar la colmatación de la industria en Beniparrell entorno al Camí Reial y también el salto cuantitativo de instalaciones entre la vía férrea y la nueva V-21, siguiendo la tendencia de los municipios al norte de urbanizar esta 

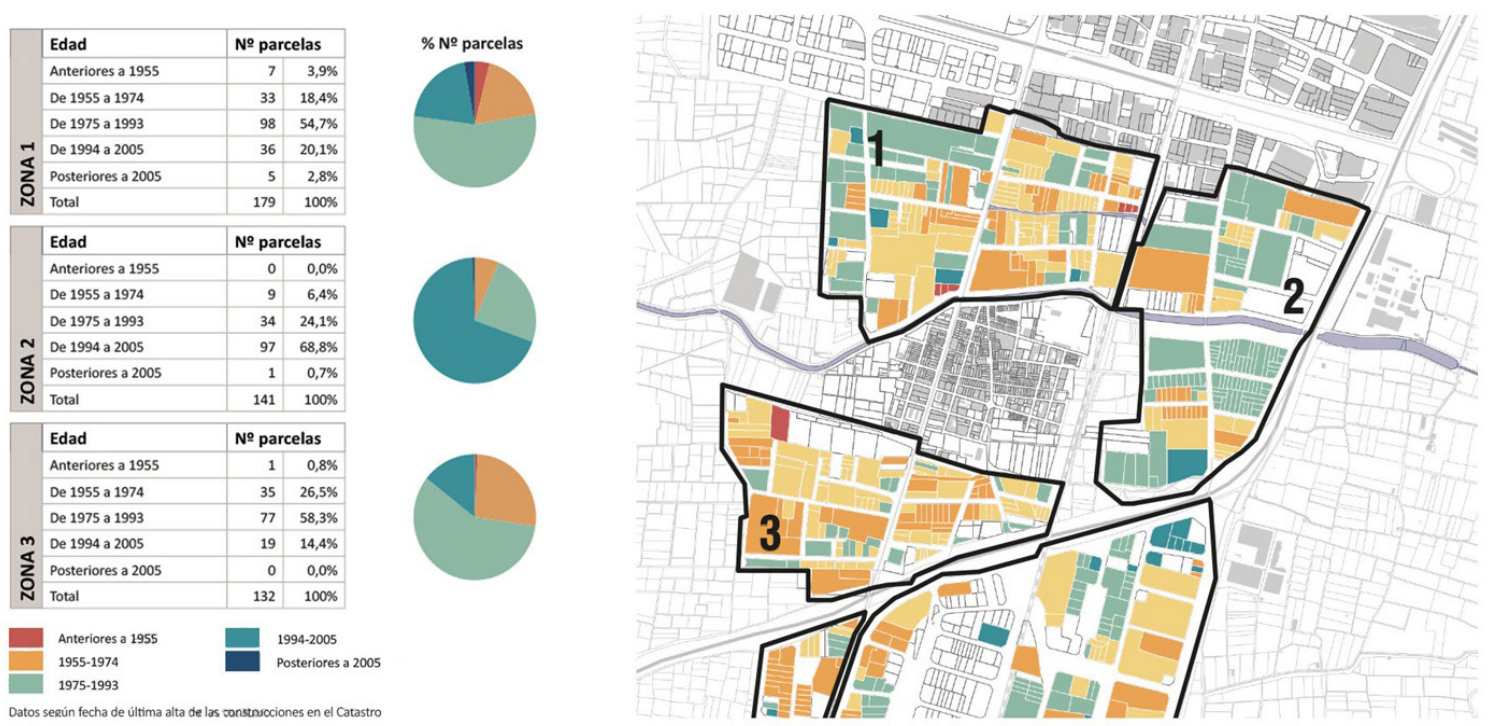

Fig. 2. Edad de la edificación (Fuente: (Cano Hurtado et alt, 2018))

banda. Esta tendencia continuará en los años posteriores hasta llegar en la actualidad a un territorio consolidado y saturado en el que el núcleo residencial ha quedado embebido por el tejido industrial. Sólo el barranco, estrangulado por la urbanización, la vía del tren y algunos terrenos agrícolas hacen de colchón entre industria y residencia.

\section{Beniparrell: actividad económica y relaciones actuales}

En el marco del Taller de Arquitectura y La actividad económica en Beniparrell ha venido marcada tradicionalmente por la industria del mueble. Esta actividad, muy presente a lo largo de la Pista de Silla, encontró aquí un eje de desarrollo de importancia regional. No es de extrañar, por tanto, que la industria de Beniparrell tuviera una repercusión e inversión mucho más extensa que su propio término municipal. Se trata de un ejemplo paradigmático, dada la gran proporción de suelo industrial que posee y su cercanía al núcleo urbano. El área residencial ocupa tan solo un $9 \%$ de su superficie, mientras que el suelo dedicado a actividades económicas es cuatro veces mayor, un $36 \%$. Beniparrell cuenta con $3,7 \mathrm{~km} 2$ y menos de 2.000 habitantes en 2018 . Su ubicación privilegiada para la localización de industria, con unas distancias a la autovía más próxima de $1 \mathrm{~km}(\mathrm{~V}-31, \mathrm{~A}-7), 20 \mathrm{~km}$ al aeropuerto y $11 \mathrm{~km}$ al puerto potenciaron la industrialización de su territorio.

La actividad económica del municipio se concentra en seis polígonos diferenciados (ver tabla 1 y Figura 3) pero contiguos, dispuestos en torno al núcleo residencial. Sin embargo, aunque conformen un continuo edificado, es destacable la poca conexión entre ellos, siendo el Camí Real el que asume estas relaciones de escala local en la mayoría de los casos. Pero, además en una escala supramunicipal, pese que en la actualidad la V-21 asume las circulaciones territoriales, el Camí Real sigue siendo la vía articuladora entre los asentamientos industriales de Beniparrell y los municipios colindantes. Este hecho hace que el tráfico pesado siga siendo protagonista diario en este eje y quede en muchos momentos saturado. Cabe destacar, en este sentido, que este eje es, además, el origen del desarrollo residencial del municipio y actual calle principal, por lo que la importancia estructural y cultural de esta vía es clave para la reconsideración de nuevas estructuras y relaciones urbanas.

Por otro lado, la actividad del mueble antes mencionada ha ido perdiendo peso, dejando atrás naves vacías y obsoletas que por su tipología o dimensiones pueden dificilmente albergar otras actividades. La mayoría de las parcelas están entre los 500 y los $5.000 \mathrm{~m} 2$ (ver Figura 4) y la implantación de estas es desordenada, ya que heredan la parcelación 


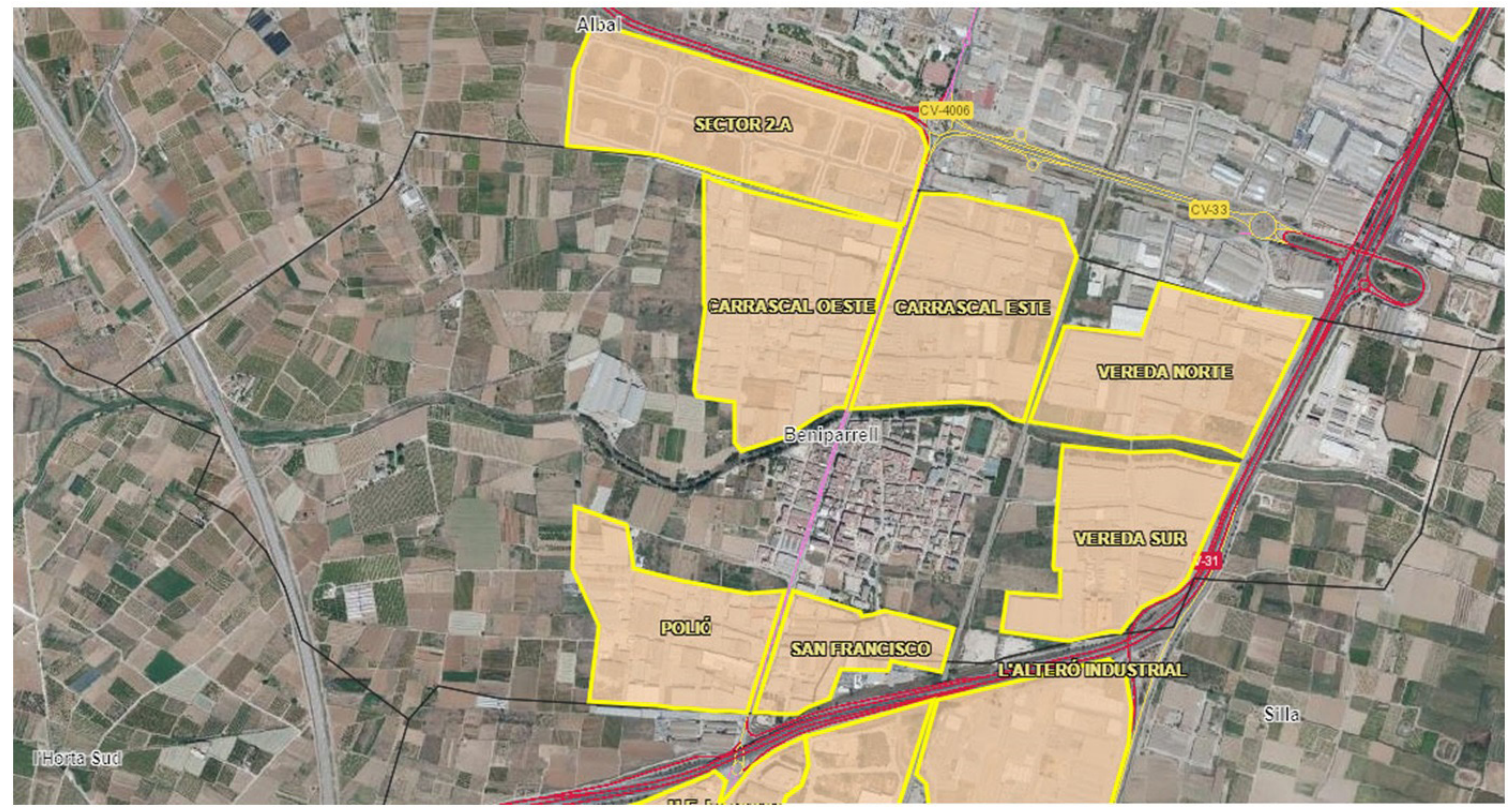

Fig. 3. Municipio de Beniparrell con sus áreas industriales resaltadas. (Fuente: Mapa de suelo industrial de la CV (IVACE, 2019))

\begin{tabular}{|c|c|}
\hline DENOMINACIÓN DEL POLÍGONO & SUPERFICIE (m2) \\
\hline Polió & $225.850,00$ \\
\hline San francisco & $86.589,00$ \\
\hline Vereda sur & $175.253,00$ \\
\hline Vereda norte & $222.560,00$ \\
\hline Carrascal este & $200.930,00$ \\
\hline Carrascal oeste & $271.817,00$ \\
\hline Total & $1.182 .999,00$ \\
\hline
\end{tabular}

Tab. 1. Superficie de las áreas industriales de Beniparrell. (Fuente: Mapa de suelo industrial de la CV (IVACE, 2019 )) agrícola previa y se desarrollan sin ningún tipo de planificación previa.

El espacio público de estos polígonos tampoco aporta calidad alguna a estas áreas, ya que ni a nivel de instalaciones, en ocasiones inexistentes, ni en cuanto a calidad urbana ofrecen ningún atractivo para la instalación de nuevas empresas. Además, el mantenimiento de las instalaciones y del espacio público ha sido escaso, por lo que la realidad construida no posee ni calidad ni los servicios mínimos requeridos.
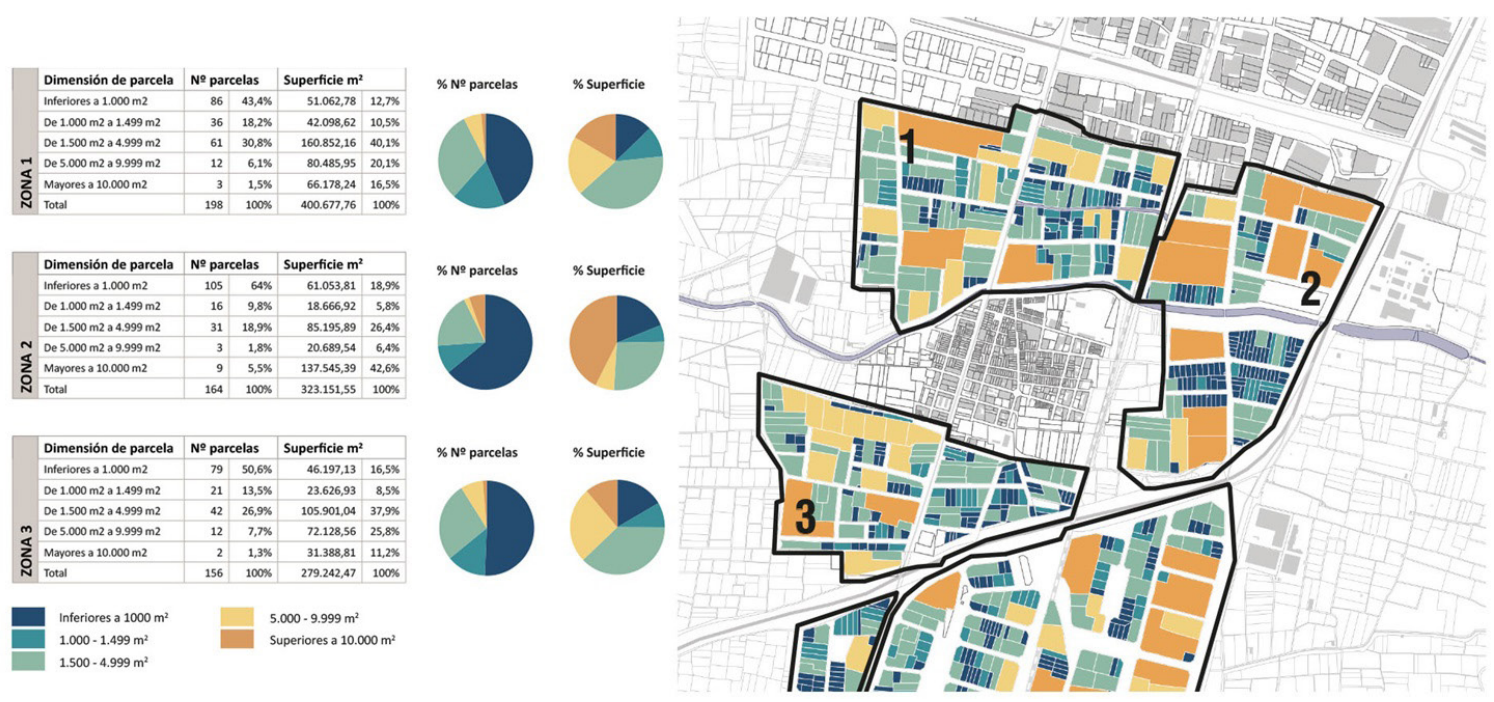

Fig. 4. Tamaño de parcelas (Fuente: (Cano Hurtado et alt. 2018)) 


\section{Pautas para una nueva relación industria- residencia}

En los últimos tiempos es evidente la evolución de la industria hacia nuevas actividades económicas, más alejadas de los efectos nocivos de los siglos pasados $\mathrm{y}$, por tanto, más compatibles con otros usos, como el residencial. La terciarización de la economía, sobre todo en áreas metropolitanas es un hecho al que los tejidos industriales todavía no se han adaptado. Como afirma Paz Benito (Benito, 2005). "La ciudad, reinventada a sí misma en etapas sucesivas de expansión y cambio, al igual que el propio sector industrial, encuentra en las iniciativas de recuperación y puesta en valor del patrimonio industrial, así como en las operaciones de regeneración y rehabilitación de espacios productivos degradados, en el planeamiento urbanístico y la ordenación del territorio, en las nuevas tecnologías y en los nuevos modos de producir y de trabajar fórmulas e instrumentos que perpetúan una relación antigua y estructural, aunque en metamorfosis permanente." Es por ello necesario reflexionar, desde la disciplina urbanística, sobre estos cambios socio-económicos para incorporarlo a las planificaciones urbanas y a las operaciones de regeneración y modernización de las áreas de actividad económica. (Hatuka 2017)

En el caso de Beniparrell, además, nos encontramos en un área industrial ya consolida $\mathrm{y}$ con necesidad de regeneración, y por ello ante la oportunidad de crear relaciones ventajosas industria-residencia en ámbitos donde hasta ahora, por falta de regulación y planificación chocaban dos realidades muy diferentes. Si bien es cierto que las instalaciones industriales no se encuentran dentro del casco urbano residencial, la colmatación del suelo industrial en el municipio ha dado lugar a un continuo urbanizado sin espacios de transición ni relaciones. Sólo el barranco al norte, estrangulado por la urbanización, y las vías del tren se han convertido en límites físicos entre ambas realidades.

El libro de Renovación urbana y actividad económica (Cano Hurtado, et alt. 2018) estudia el conjunto formado por las áreas de actividad económica conformadas por los municipios de Silla, Beniparrell y Alcàsser. La fase de análisis se completa con una definición de estrategias CAME (corregir, afrontar, mantener y explotar) propuestas como respuesta al DAFO en el municipio de Beniparrell. Las estrategias se organizan en seis bloques: Primero, vinculado a los ejes territoriales y los accesos. Segundo, destinadas a incorporar la infraestructura verde dentro de la dinámica del proyecto. La tercera, agrupa los objetivos relacionados con la estructura viaria como elemento soporte de las actividades. En cuarto lugar, se establecen los criterios generales sobre los modos de transporte y la movilidad sostenible. La quinta estrategia, articularía los objetivos relativos a los usos del suelo y las condiciones y criterios de parcelación. Y, por último, la última estrategia centraría sus propuestas en la señalética, tan importante en este tipo de espacios, y las condiciones

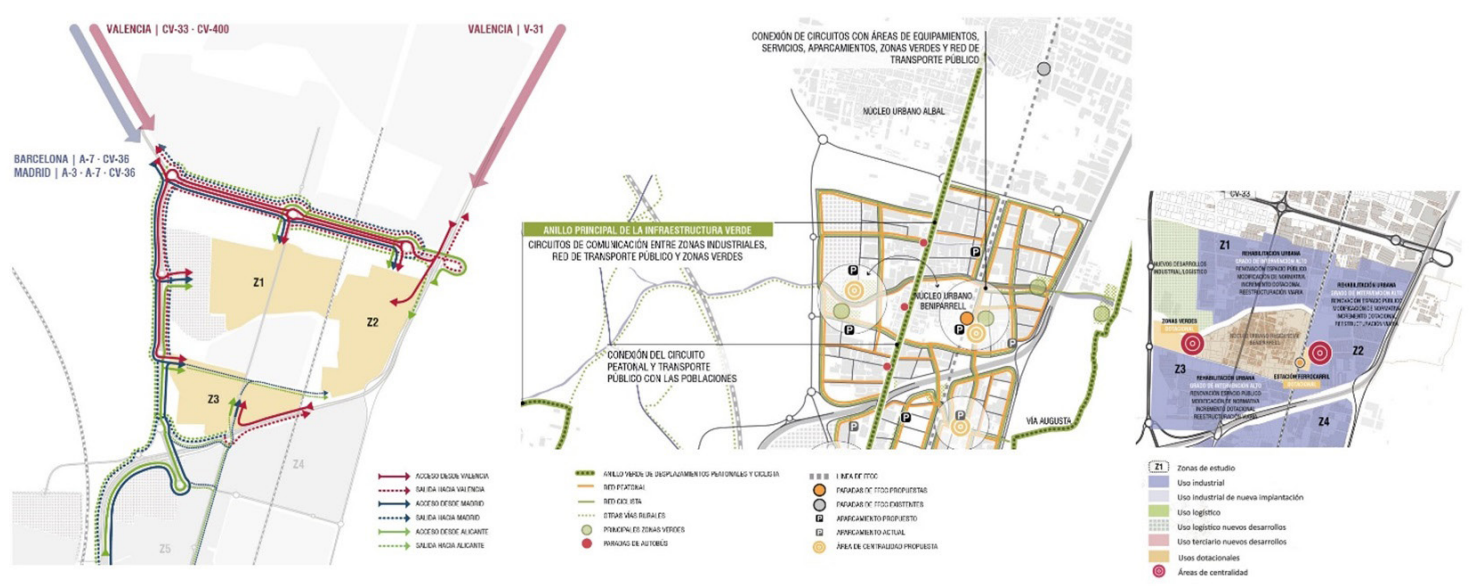

Fig. 5. Tamaño de parcelas (Fuente: (Cano Hurtado et alt. 2018)) 
de imagen. Estas estrategias apuestan por soluciones que se deberían aplicar a muchas de las áreas industriales de edad similar a las de Beniparrell. Entre todas ellas, adquiere una gran importancia la relación de aquellas áreas cercanas a los núcleos urbanos, como objetivo fundamental para mejorar fomentar la relación del espacio urbano-residencial.

\section{Líneas a seguir en Beniparrell para nuevas relaciones industria-residencia:}

De un modo más concreto se establecen las líneas prioritarias para llevar a cabo en el municipio actuaciones que mejoren las áreas industriales y las relaciones entre la residencia y la industria. (Figura 5).

- Se propone la mejora en los accesos a las áreas industriales, puesto que actualmente se producen principalmente por el Camí Reial lo que provoca colapsos y saturación. Unos nuevos puntos de acceso a estas áreas permitiría descongestionar esta vía y ofrecer alternativas, de forma que el área residencial no estuviera tan condicionada al paso de tráfico pesado.

- Establecer el Camí Reial como un eje cultural, creando un nuevo paisaje urbano y oportunidades para localización de nuevas empresas que buscan una imagen de calidad y representativa . La accesibilidad alternativa a las zonas industriales permitiría convertir este eje en un verdadero espacio de oportunidad, menos condicionado por el tráfico pesado $\mathrm{y}$ más orientado a convertirse en un elemento articulador, de calidad y representativo de todo el suelo urbano.

- Incorporar la Infraestructura verde como herramienta clave en la creación de nuevas relaciones. Se debe integrar y potenciar en las distintas escalas, en el contexto territorial y urbano así como en la calidad del espacio público.

- Identificar los bordes urbanos naturales. Revalorizar el barranco de Picassent puede convertirlo en un elemento vertebrador esteoeste, formando parte de la infraestructura verde territorial al conectarlo con el Parque Natural de la Albufera y la Sierra del Ave. De la misma manera, el tratamiento del como eje que configure en su entorno paseos peatonales y ciclistas trascienden el ámbito de proyecto, alcanzando las entidades metropolitanas próximas.

- En la recomposición del área será imprescindible tener en cuenta las circulaciones y secciones viarias. Actualmente, las circulaciones no disponen de jerarquías, y en muchos casos, las tramas estan inacabadas, no poseen aparcamientos y en algunos casos ni siquiera aceras o calzada en condiciones. Una nueva jerarquía viaria, acorde con las necesidades actuales y con las dimensiones adecuadas ofrecerían una estructura urbana de calidad capaz de acoger nuevas actividades.

- Mayor relación con el transporte público e implantación de una movilidad sostenible. Las conexiones actuales de Beniparrell con núcleos cercanos y con Valencia son escasas y obligan a la utilización del vehículo privado. Las condiciones del entorno, la proximidad con muchos otros asentamientos urbanos y su ubicación en el área metropolitada de Valencia permiten y a la vez requieren de una reflexión sobre la movilidad en términos de sostenibilidad.

- Recomposición del tejido urbano para crear una nueva estructura urbana, más flexible $\mathrm{y}$ acorde a los nuevos usos y vinculada con la nueva estructura y jerarquía viaria.

- Revisión de los usos urbanos y las "calles frontera", para tratarlos como elementos de articulación con otras tramas urbanas próximas o con el espacio libre metropolitano de carácter finalista (corredores ambientales, espacios protegidos, etc.)

- Revisión de la edad de la edificación: el entorno del casco urbano tiene más de 40 años. La vida útil de un edificio industrial está en torno a una media de 20 años, por lo que se puede decir que el parque de edificios está más que obsoleto. Esto da más razones, si cabe, a pensar que es necesaria una intervención. (ver Figura 2)

- Revisión del parcelario: junto a la obsolescencia de la edificcación, se observa también que la morfología y tamaños de parcela, con origen en la trama agrícola, no son los adecuados para la impltación de la nueva industria que necesita espacios y requisitos distintos a los presentes.

- Dotaciones y servicios urbanos: la contigüidad de las AAE con el casco urbano 
ofrece a éstas la oportunidad de compartir servicios y equipamientos. Las áreas industriales no poseen en este momento ni dotaciones ni servicios, por lo que la necesaria reforma es una oportunidad para resolvar este problema. La mejora de las relaciones viarias entre ambas realidades permitirá un uso compartido de estos recursos, lo que es además más sostenible, y permitirá una mezcla de usos. Para concluir, cabe destacar que más allá del tamaño o relación entre la industria y la residencia, así como las actuaciones que deban llevarse a cabo en las áreas industriales para llevar a cabo su regeneración, la implicación de los distintos agentes, privados o públicos, y su capacidad de concertación entorno a proyectos concretos y consensuados es un punto clave. (Caravaca and Méndez 2003).

\section{Referencias}

Benito del Pozo, Paz. "Pautas actuales de la relación entre industria y ciudad." Ería: Revista cuatrimestral de geografía, no. 66, 2005, pp. 57-70, http://dialnet.unirioja.es/ servlet/oaiart?codigo $=1281979$.

Burdett, Richard, Andreu Domingo, Zaida Muxí, Sebastià Sarasa, Sebastià Jornet, Carles Llop, Néstor Duch, Antonio Font, Miquel Morell, and Amador Ferrer. 2014. Urbanisme i Noves Dinàmiques Socials i Productives: Workshop 3, 28-5-2014= Urbanismo y Nuevas Dinámicas Sociales y Productivas $=$ Urbanism and New Dynamics of Society and Production. Urbanismo y Nuevas Dinámicas Sociales y Productivas. Barcelona: Barcelona : Àrea Metropolitana de Barcelona, D.L. 2014.

Caravaca, Inmaculada, and Ricardo Méndez. 2003. Trayectorias Industriales Metropolitanas: Nuevos Procesos, Nuevos Contrastes. Eure-Revista Latinoamericana De Estudios Urbano Regionales - EURE. Vol. 29. https://doi.org/10.4067/S025071612003008700003.

Cano Hurtado, Juan J., Blasco Sánchez, Carmen. 2002. El Espacio Industrial en la Comunidad Valenciana. Valencia: Editorial Universitat Politécnica de València.

Cano Hurtado, Juan J., Blasco Sánchez, Carmen, Martínez Pérez, Francisco J.,
Gascón Hernández, Ana, y Esteve Aguado, Carlos. 2018. Renovación urbana y actividad económica. Propuesta de intervención territorial en un entorno territorial estratégico. ISBN: 978-84-9048-757-0. Valencia: Editorial Universitat Politècnica de València.

De Terán, Fernando, 1972. "Descongestión industrial, zonificación y urbanismo: Experiencias extranjeras y en España". En 3 Congreso Nacional Iranor: Dirección de empresas y cambios tecnológicos. Madrid, (23-36).

Esteban I Noguera, Juli. 2007. «Els espais d'activitats econòmiques en el planejament territorial». Papers: Regió Metropolitana de Barcelona: Territori, estratègies, planejament, 45, 56-63.

Generalitat Valenciana. 2014. Estrategia de política industrial de la Comunitat Valenciana-Visión 2020.

Hatuka, Tali. 2017. Industrial Urbanism: Typologies, Concepts and Prospects. Built Environment. Vol. 43. https://doi. org/10.2148/benv.63.3.10.

Institut Catalá del Sòl. 2007. Sectores de Actividad Económica. Libro de estilo. Barcelona: Institut Catalá del Sòl.

IVACE. (Institut Valencià de Competitivitat Empresarial) Mapa Suelo Industrial de la Comunitat Valenciana. (http://www.ivace. es/visor_pol/)

López Groh, Francisco. 2011. La regeneración de áreas industriales. Madrid: Sepes Entidad Estatal de Suelo.

Mancomunitat Intermunicipal de L'Horta Sud. Plan Estratégico Comarcal de L'Horta Sud 2018-2022. Volumen I. 2019. [http://mancohortasud.es/wp-content/ uploads/2019/01/Plan-Estrategico-HortaSud-2018-2022.pdf]

Muñoz Criado, Aránzazu, y Vicente Doménech Gregori. 2011. Actividad económica: objetivo 24: prever suelo para la actividad económica en cantidad suficiente y adecuada. Valencia: Generalitat Valenciana. Conselleria de Medi Ambient, Aigua, Urbanisme i Habitatge, 2011. 